\title{
ON THE PERFORMANCE OF MULTICARRIER CDMA USING MULTIPLE TRANSMITTERS
}

\author{
Quentin H. Spencer G A. Lee Swindlehurst \\ Department of Electrical \& Computer Engineering \\ Brigham Young University \\ Provo, Utah 84602 \\ spencerq@ee.byu.edu
}

\begin{abstract}
Recent research has demonstrated the existence of transmission schemes and codes which provide diversity gain by using multiple transmitting antennas. This paper applies the concepts of multicarrier DS-CDMA systems to the multiple transmitter case. The multicarrier CDMA system discussed here was originally based on the assumption that the frequency-selective fading was uncorrelated from one subcarrier to the next. In this paper we derive the performance for a system whose subcarriers are subject to correlated fading, and the performance of a system partially or completely decorrelated by distributing the subcarriers across transmit antennas.
\end{abstract}

\section{INTRODUCTION}

The increasing demand for bandwidth in wireless communications systems has resulted in numerous systems which use multiple antennas. Initially, this was limited to systems with multiple receivers, which employed diversity combining or beamforming methods to improve signal to noise ratio. More recently, attention has turned toward systems that use multiple transmitters, particularly in light of the discovery that, under the right conditions, a multiple input multiple output (MIMO) channel can achieve capacity increases on the order of the array size. Some of the proposed transmission schemes which promise the greatest performance gains involve data processing that is highly coordinated between the transmitter and receiver, requiring knowledge of the channel at both ends. Other schemes, while more limited in their potential performance gains, achieve diversity gains through so-called "space-time" coding methods which are independent of the channel and the specific configuration of the receiver. In this paper we explore one such scheme which combines the concepts of transmit diversity and multicarrier CDMA.

The original idea behind multicarrier CDMA was to create a broadband CDMA system that would have greater robustness to some types of fading and interference than a single carrier system occupying the same bandwidth. The scheme we use as our model is that proposed by Kondo and Milstein [1]. In their approach, the frequency band is

This work was supported by the National Science Foundation under Wireless Initiative Grant CCR 99-79452.

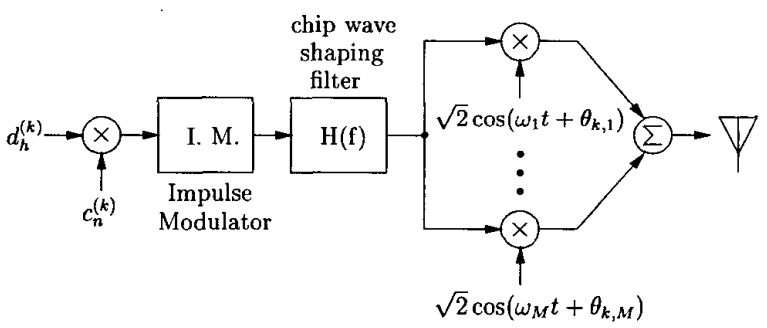

Figure 1: Diagram of the transmission scheme in [1]

broken into $M$ sub-bands. Within each band the fading is assumed to be approximately flat, and uncorrelated with neighboring frequency bands. The data to be transmitted is spread using a new spreading code whose chip rate is reduced by a factor of $M$ to fit the bandwidth allocated to the subcarriers. This signal is then modulated onto $M$ separate carriers, and these signals are all summed together to be transmitted. An illustration of this is shown in Figure 1. In the diagram, $d_{h}^{(k)}$ is the data to be transmitted for the $k^{\text {th }}$ user, and $c_{n}^{(k)}$ is the corresponding chip sequence.

The receiver for this system separately demodulates and despreads each subcarrier. At the output of the matched filter, the signals are combined using maximal-ratio combining. Under the conditions described, this system is robust to narrowband interference or fading of individual subcarriers because of the fact that identical signals are transmitted on each subcarrier. If one signal is lost, all of the information in the signal is still contained in the other subcarriers.

In this paper, we first discuss how this scheme can be applied to multiple transmit antennas. Second, we derive the performance of a multicarrier CDMA system in the presence of correlated fading. Using these theoretical results, we present numerical results to compare the performance of the system in different types of fading, and show the performance improvements achievable by using multiple antennas. 


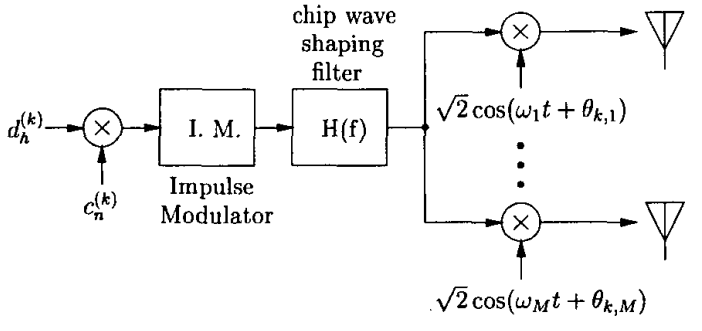

Figure 2: Block diagram of multicarrier applied to multiple transmitters

\section{MULTIPLE ANTENNA TRANSMISSION SCHEME}

In an environment where the fading is correlated from one subcarrier to the next, decorrelation can be achieved by transmitting the subcarriers from separate antennas, as illustrated in Figure 2. Instead of being summed as in Figure 1 , each modulated narrowband CDMA signal is sent to a different antenna. If the different spatial channels are independent, the fading characteristics of each subcarrier are uncorrelated. The performance of this system will be equivalent to the performance of the original system in uncorrelated fading. The other benefit of this scheme is that the decorrelation effect of putting the subcarriers on separate antennas removes the upper bound on the number of carriers. This upper bound was due to the fact that in order for a single antenna multicarrier system to have independent fading for each carrier, the bandwidth of a subcarrier must be less than or equal to the coherence bandwidth of the channel.

To quantify the performance gain of multiple transmit antennas in correlated fading, we parameterize the correlation between the channel gain for one carrier and its neighbors as follows. Let $r_{i}$ be the complex gain of the channel for the $i^{\text {th }}$ subcarrier, and define $\mathbf{r}=\left[\begin{array}{ll}r_{1} & r_{2}, \ldots r_{M}\end{array}\right]^{T}$, so that the overall channel covariance matrix is $\mathbf{R}_{r}=E\left[\mathbf{r r}^{\mathbf{T}}\right]$. Assume that for the single antenna system the correlation between any two subcarriers is $E\left[r_{i} r_{i-n}\right]=\xi^{n}$, so that

$$
\mathbf{R}_{\mathbf{r}}=\left[\begin{array}{cccc}
1 & \xi & \ldots & \xi^{M-1} \\
\xi & 1 & \ldots & \xi^{M-2} \\
\vdots & \vdots & \ddots & \vdots \\
\xi^{M-1} & \xi^{M-2} & \ldots & 1
\end{array}\right]
$$

Note that in this case $\mathbf{R}_{\mathbf{r}}$ is a symmetric Toeplitz matrix.

When there are $M$ subcarriers and $M$ transmitters whose fading characteristics are uncorrelated, $\mathbf{R}_{\mathbf{r}}$ becomes the identity. If there are $Q<M$ transmitters, the best we can do is to rotate the carrier allocations among the transmitters in such a way as to make any subcarriers on the same antenna minimally correlated. This is done by assigning subcarriers $1, Q+1,2 Q+1$, etc. to the first antenna, subcarriers $2, Q+2,2 Q+2$, etc. to the second antenna, and so on, so that the $j^{\text {th }}$ carrier is assigned to antenna number $j \bmod Q$. This is illustrated in Figure 3 for the case of three antennas and eight subcarriers. In this example, the

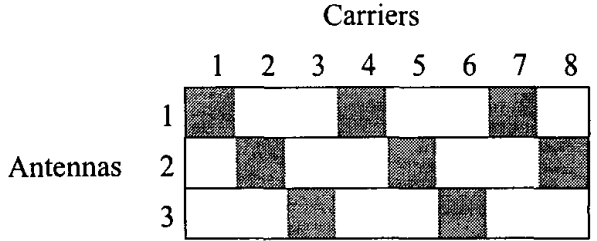

Figure 3: Example of frequency allocation for 3 antennas and 8 carriers

first row of $\mathbf{R}_{\mathbf{r}}$ will be

$$
\left[\begin{array}{llllllll}
1 & 0 & 0 & \xi^{3} & 0 & 0 & \xi^{6} & 0
\end{array}\right] \text {. }
$$

In general, for $Q$ antennas, only every $(n Q)^{\text {th }}$ off-diagonal will be nonzero for this carrier allocation scheme.

The covariance matrix can be further generalized to include possible correlations between the channel gains associated with each antenna. Ideally, antennas in a diversity system should be spaced far enough apart so that their fading characteristics are uncorrelated. However, there are situations where this is not possible, particularly in diversity applications for mobile communications. If we define the correlation between channel gains for array elements $k$ and $l$ to be $\zeta_{k l}$, then in general

$$
R_{r}(i, j)=\xi^{|i-j|} \zeta_{(i \bmod Q),(j \bmod Q)},
$$

and the structure of $\mathbf{R}_{\mathbf{r}}$ is no longer Toeplitz.

Given the covariance matrix $\mathbf{R}_{\mathbf{r}}$, the problem of finding the bit error rate performance of any of these systems is now reduced to solving for the probability distribution of the output of a maximal ratio combiner with arbitrarily correlated inputs.

\section{PROBABILITY OF ERROR FOR THE MAXIMAL-RATIO COMBINER WITH CORRELATED FADING}

The problem of finding error rates for a maximal-ratio combiner in correlated fading has been addressed by Zhang [2], who solved the problem for Nakagami fading. Rayleigh fading is a special case of Nakagami fading, so the derivation is very similar, and some of the results are quoted here.

We begin with the result in [1] that the signal to noise ratio of the system is:

$$
\rho_{m}=N^{2} E_{c} \gamma
$$

where $E_{c}$ is the energy per chip, $N$ is the number of chips per symbol, and

$$
\gamma=\sum_{k=1}^{M} \frac{\alpha_{1, k}^{2}}{\sigma_{k}^{2}}=\sum_{k=1}^{M} q_{k},
$$

where $\alpha_{1, k}$ is the gain of the $k^{\text {th }}$ carrier for the first user, and $\sigma_{k}^{2}$ is its variance. As a result, each $q_{i}$ is an exponentially distributed random variable with the following PDF:

$$
f_{q}\left(q_{i}\right)=\sigma_{i}^{2} e^{-\sigma_{i}^{2} q_{i}} .
$$


At this point, in [1] the $q_{i}$ are i.i.d., so the PDF of the sum is found by taking a product of the characteristic functions. For the correlated case the characteristic function is a product, but related more indirectly to the characteristic functions of the individual random variables. We quote the following steps from [2]. The joint characteristic function of the instantaneous SNR for an L-branch maximal ratio combining system is:

$$
\begin{aligned}
\phi_{\mathbf{r}}\left(j t_{1}, \ldots, j t_{L}\right) & =E\left[e^{j \sum_{k=1}^{L} \gamma_{k} t_{k}}\right] \\
& =\operatorname{det}(\mathbf{I}-j \mathbf{T} \boldsymbol{\Gamma})^{-m}
\end{aligned}
$$

where

$$
\mathbf{T}=\left[\begin{array}{lll}
t_{1} & & \mathbf{0} \\
& \ddots & \\
\mathbf{0} & & t_{L}
\end{array}\right],
$$

$m$ is the Nagakami fading parameter, and the matrix $\boldsymbol{\Gamma}$ is related to the branch covariance matrix $\mathbf{R}_{\mathbf{r}}$. If $\Gamma(k, l)$ and $R_{r}(k, l)$ are the $k, l^{\text {th }}$ elements of $\Gamma$ and $\mathbf{R}_{\mathbf{r}}$, the relationship can be proven to take the following form:

$$
\Gamma(k, l)=\sqrt{\frac{R_{r}(k, l)}{m}} .
$$

Rayleigh fading is the special case where the Nakagami parameter $m=1$, so $\Gamma(k, l)=\sqrt{R_{r}(k, l)}$.

The determinant can now be expressed as a product:

$$
\begin{aligned}
\Phi_{\gamma}(s) & =|\mathbf{I}-s \boldsymbol{\Gamma}| \\
& =\prod_{k=1}^{L}\left(1-s \lambda_{k}\right)^{-1} .
\end{aligned}
$$

In order to derive the associated PDF, the product must be converted into a sum, by partial fraction expansion. In its most general form, it is:

$$
\Phi_{\gamma}(s)=\sum_{k=1}^{L} \frac{\beta_{k}}{\left(1-s \lambda_{k}\right)^{r_{k}}} .
$$

This characteristic function contains the function $\Phi_{x}(s)=$ $(1-\lambda s)^{-r}$, which is the characteristic function of a gamma distribution with parameters $\lambda$ and $r$. The gamma distribution is a generalized case of the $\chi^{2}$ distribution, and has the following PDF:

$$
p_{\gamma}(x \mid \lambda, r)=\frac{1}{\lambda \Gamma(r)}\left(\frac{\gamma}{\lambda}\right)^{r-1} e^{-\frac{\gamma}{\lambda}}
$$

Given the PDF $p_{\gamma}(\gamma)$, the error probability of the multicarrier CDMA system is given by the following integral:

$$
P_{e}=\int_{0}^{\infty}\left[\frac{1}{\sqrt{2 \pi}} \int_{-\infty}^{-\sqrt{N^{2} E_{c} \gamma}} e^{-\frac{t^{2}}{2}} d t\right] p_{\gamma}(\gamma) d \gamma
$$

To solve this integral, we use the linearity property of the Fourier transform to state the PDF of $\gamma$ as a sum of PDFs of the form found in (14):

$$
p_{\gamma}(\gamma)=\sum_{k=1}^{L} \frac{\beta_{k}}{\lambda_{k} \Gamma\left(r_{k}\right)}\left(\frac{\gamma}{\lambda_{k}}\right)^{r_{k}-1} e^{-\frac{\gamma}{\lambda_{k}}} .
$$

Substituting this into the integral:

$$
\begin{aligned}
& P_{e}= \int_{0}^{\infty} \int_{-\infty}^{-\sqrt{N^{2} E_{c} \gamma}} \frac{1}{\sqrt{2 \pi}} e^{-\frac{t^{2}}{2}} \\
& {\left[\sum_{k=1}^{L} \frac{\beta_{k}}{\lambda_{k} \Gamma\left(r_{k}\right)}\left(\frac{\gamma}{\lambda_{k}}\right)^{r_{k}-1} e^{-\frac{\gamma}{\lambda_{k}}}\right] d t d \gamma } \\
&= \sum_{k=1}^{L} \frac{\beta_{k}}{\sqrt{2 \pi}}\left[\int_{0}^{\infty} \int_{-\infty}^{-\sqrt{N^{2} E_{c} \gamma}} \frac{1}{\left(\frac{\gamma}{\lambda_{k} \Gamma\left(r_{k}\right)}\right.} e^{r_{k}-1} e^{-\frac{t^{2}}{2}-\frac{\gamma}{\lambda_{k}}} d t d \gamma\right] \\
&= \sum_{k=1}^{L} \frac{\beta_{k}}{2}\left[1-\frac{\sqrt{2 \lambda_{k} N^{2} E_{c}}}{\sqrt{\pi}} \frac{\Gamma\left(r_{k}+1 / 2\right)}{\Gamma\left(r_{k}\right)}\right. \\
&\left.{ }_{2} F_{1}\left(\frac{1}{2}, L+\frac{1}{2} ; \frac{3}{2} ;-\frac{1}{2} \lambda_{k} N^{2} E_{c}\right)\right]
\end{aligned}
$$

where ${ }_{2} F_{1}(a, b ; c ; x)$ is the generalized hypergeometric function. This expression for error probability has two special cases. First, when the covariance matrix is identity, all values of $\beta_{k}$ are zero except the last one, where $r_{L}=L$ and $\beta_{L}=1$. Second, when the eigenvalues are all unique, $r_{k}=1$ and the hypergeometric function simplifies so that

$$
P_{e}=\sum_{k=1}^{L} \frac{\beta_{k}}{2}\left[1-\sqrt{\frac{\lambda_{k} N^{2} E_{c}}{2+\lambda_{k} N^{2} E_{c}}}\right] .
$$

\section{COMPARISON OF RESULTS FOR CORRE- LATED AND UNCORRELATED FADING}

In order to evaluate the error probability and compare the results for correlated and uncorrelated fading, we use the same system parameters as those defined in [1]. To summarize, the signal spectra of the individual carriers do not overlap, and the chip waveform is shaped using a raisedcosine filter with parameter $\alpha$. When there are $K$ users, the system has noise variance

$$
\frac{K-1}{2 N}\left(1-\frac{\alpha}{4}\right)+\frac{N \eta_{0}}{2}
$$

and the conditional SNR of the system is

$$
\begin{aligned}
\rho_{m} & =N^{2} E_{c} \sum_{i=1}^{M} \frac{\alpha_{1, i}^{2}}{\sigma_{i}^{2}} \\
& =\left[\frac{K-1}{2 N}\left(1-\frac{\alpha}{4}\right)+\frac{\eta_{0}}{2 N E_{c}}\right]^{-1} \sum_{i=1}^{M} \alpha_{1, i}^{2} .
\end{aligned}
$$

For the results presented here, we assume that $\alpha=0.5$.

Figure 4 shows a comparison of various antenna configurations for a system with 4 carriers. We assume that the fading correlation between antennas is parameterized by a single value $\zeta$, where the channel gains for two antennas $g_{i}$ and $g_{j}$ are related by the covariance function $E\left[g_{i} g_{j}\right]=\zeta^{|i-j|}$. In the figure we compare antennas with channel gains that have correlation parameter $\zeta=0$ and 0.5. All of the curves assume a value of $\xi=0.5$ for the channel correlation function in the frequency domain. The 


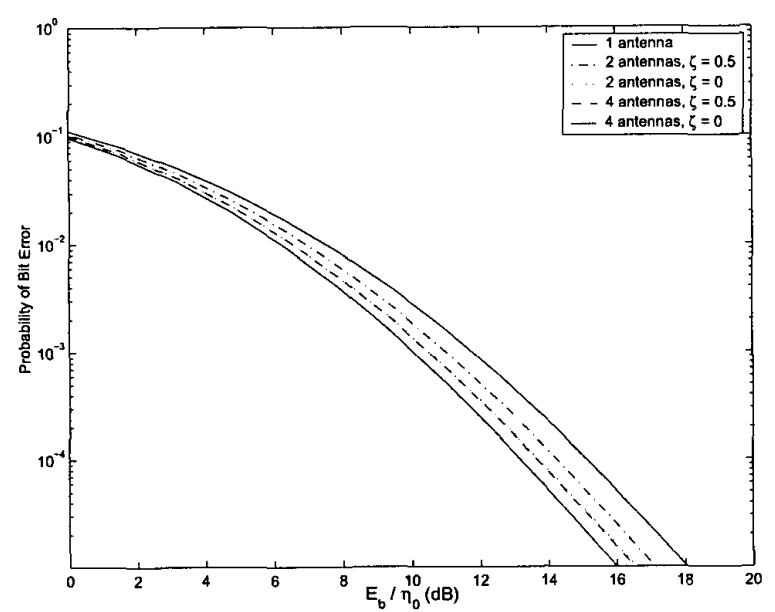

Figure 4: BER vs. SNR for 4 carriers, $\alpha=0.5, \xi=0.5$ and varying antenna configurations

top curve is for a single antenna. The second is for two antennas with $\zeta=0.5$. The third and fourth, which are virtually indistinguishable, are for two antennas, $\zeta=0$, and four antennas, $\zeta=0.5$. This implies that using four closely spaced antennas would have the same performance as only two antennas that were more widely spaced. The last curve is for four antennas, with no correlation. This is the same as the performance curve for four subcarriers on a single antenna system with no intercarrier fading correlation.

For comparison with the results from [1], Figures 5 and 6 duplicate the original results for uncorrelated fading, and show the performance curves for channels whose frequency domain fading correlation is parameterized by $\xi=0.25$ and 0.5 . Figure 5 is for a single user channel, and Figure 6 is for 50 co-channel users. The curves for uncorrelated fading $(\xi=0)$ could be interpreted as either a single antenna system in a fading environment that is uncorrelated, or a multiple antenna system in a fading environment that would have been correlated using one antenna, but has achieved decorrelation of the subcarriers by sufficient separation of the antennas. In fading environments with $\xi=0.5$ at high SNR, there is a gain of approximately 1-2 dB achieved by decorrelating the subcarriers, and significantly more in multiuser channels. Also worth noting is the performance gap between two uncorrelated carriers and four highly correlated carriers. Though the gap is more significant with only one user, it is still present with a large number of users. This illustrates the fact that even with a single antenna system, if the fading characteristics are such that increasing the number of carriers from two to four introduces correlation, there is still a performance gain to be achieved in using more carriers.

\section{CONCLUSION}

We have derived the performance of a multicarrier CDMA system when the subcarriers are subject to correlated fading. This allows a performance comparison of a variety

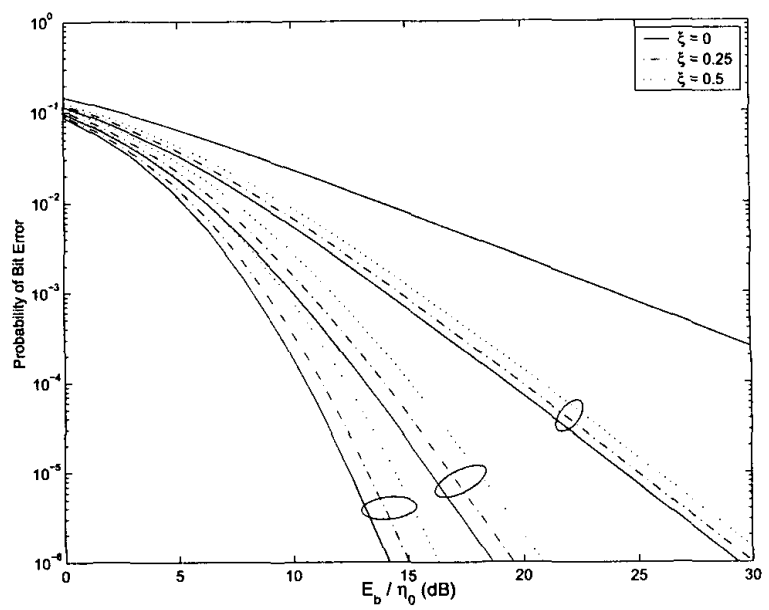

Figure 5: BER vs. SNR under different fading conditions for a single user

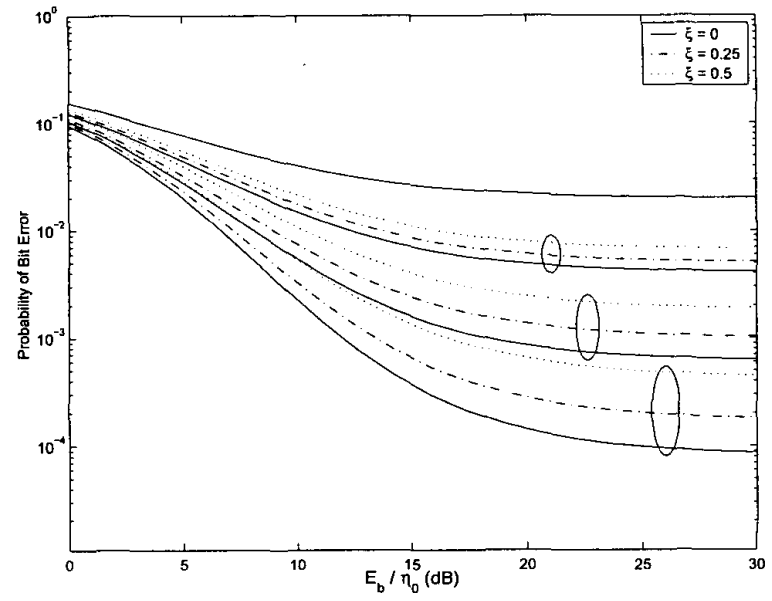

Figure 6: BER vs. SNR under different fading conditions for 50 users

of different transmission schemes under varying conditions. We propose transmitting subcarriers on separate antennas as a method to decorrelate them in correlated fading channels. This enables the use of a higher number of subcarriers, which can significantly improve performance.

\section{REFERENCES}

[1] Shiro Kondo and Laurence B. Milstein, "Performance of Multicarrier DS-CDMA Systems," IEEE Transactions on Communications, vol. 44, no. 2, pp. 238-246, February 1996.

[2] Q. T. Zhang, "Maximal-Ratio Combining Over Makagami Fading Channels With an Arbitrary Branch Covariance Matrix," IEEE Transactions on Vehicular Technology, vol. 48, no. 4, pp. 1141-1150, July 1999. 